\title{
In vitro screening of lactobacilli with antagonistic activity against Helicobacter pylori from traditionally fermented foods
}

\author{
X. Chen, ${ }^{\star 1}$ F. Tian, $\dagger^{1}$ X. Liu, $†$ J. Zhao, $†$ H.-P. Zhang, $\neq$ H. Zhang, $†$ and W. Chen ${ }^{\star 2}$ \\ *State Key Laboratory of Food Science and Technology, and \\ †School of Food Science and Technology, Jiangnan University, Wuxi 214122, P. R. China \\ $\ddagger$ Key Laboratory of Dairy Biotechnology and Engineering, Ministry of Education, Inner Mongolia Agricultural University, Hohhot, \\ Inner Mongolia 010018, China
}

\begin{abstract}
Helicobacter pylori may cause stomach diseases such as chronic gastritis, peptic ulcer, and gastric cancer, and several studies reported that lactobacilli have inhibitory effects on H. pylori. In this study, 38 Lactobacillus strains were screened for anti- $H$. pylori activity using in vitro methods, including survivability under the simulated gastric conditions, agar plate diffusion, urease activity, coaggregation, autoaggregation, and hydrocarbon analysis. The results indicate that $2 \mathrm{Lac}$ tobacillus strains showed potential anti- $H$. pylori activity in vitro. Lactobacillus plantarum 18 had the largest zone of inhibition and markedly reduced the urease activity of $H$. pylori. Lactobacillus gasseri Chen had higher coaggregation rate $(58.15 \%)$ and hydrophobicity (59.27\%) compared with the other strains. Further research is needed to verify the activities of these strains against $H$. pylori.
\end{abstract}

Key words: Lactobacillus, Helicobacter pylori, antagonism, urease activity

\section{INTRODUCTION}

Helicobacter pylori is a spiral gram-negative microorganism that causes chronic gastritis, peptic ulcer disease, and gastric cancer. The mechanisms by which $H$. pylori infection leads to gastric mucosal damage include the direct effects of virulence factors produced by $H$. pylori such as cagA, vacA, or urease; propagation and perpetuation of inflammation; oxidative stress; and induction of apoptosis in infected gastric epithelial cells (Goodwin, 1997; Graham, 1997; Dhar et al., 2003; Lee et al., 2008). At present, triple therapies that use combinations of a proton pump inhibitor (omeprazole) plus

Received May 18, 2010.

Accepted September 12, 2010.

${ }^{1}$ These authors contributed equally to this work.

${ }^{2}$ Corresponding author: weichen@jiangnan.edu.cn antibiotic (clarithromycin or amoxicillin) and metronidazole are typically used as the treatment for diseases introduced by $H$. pylori. However, problems associated with patient noncompliance and consequent relapse of H. pylori infections are common. Moreover, these drugs destroy the microenvironment in the stomach, leading to side effects. Therefore, it is desirable to search for a natural treatment against $H$. pylori infection.

Lactobacilli are natural inhabitants of the human intestinal tract and have a positive effect on bowel microecology and health. In previous studies, probiotics have been used as adjuvant therapy in the treatment of $H$. pylori; these probiotics include Lactobacillus salivarius (Kabir et al., 1997), Lactobacillus casei Shirota (Sgouras et al., 2004), Lactobacillus johnsonii La1 (Bergonzelli et al., 2006; Gotteland et al., 2008), Lactobacillus GG (Ouwehand et al., 2000; Armuzzi et al., 2001), Lactobacillus gasseri (Sakamoto et al., 2001), Bacillus subtilis (Pinchuk et al., 2001), and Bifidobacteria (Collado et al., 2005). Lactobacillus gasseri and L. casei strains have been found to decrease $H$. pylori density in the gastric mucosa (Sakamoto et al., 2001; Sgouras et al., 2004), and lactobacilli may decrease both gastric inflammation and side effects of therapy for $H$. pylori eradication (Lesbros-Pantoflickova et al., 2007). The mechanisms of anti- $H$. pylori activity of lactobacilli may be associated with decreases in urease activity and adhesive capability of H. pylori; secretion of lactic acid, bacteriocins, autolysins, and proteinaceous compounds; and suppression of $\mathrm{H}$. pylori-associated IL-8 production (Lorca et al., 2001; Kim et al., 2003; Lesbros-Pantoflickova et al., 2007). The application of lactobacilli in foodstuffs could decrease colonization of $H$. pylori in infected asymptomatic subjects, increase eradication rates when administered together with antibacterial and other drugs in symptomatic subjects, or decrease the incidence and severity of possible adverse effects due to treatments (Boyanova et al., 2009). Traditional fermented foods are important resources for isolation and screening of probiotics with health benefits. Therefore, the aim of the present study was to screen lactobacilli from tra- 
ditional fermented foods in China for in vitro activity against $H$. pylori.

\section{MATERIALS AND METHODS}

\section{Bacterial Strains}

Lactobacillus rhamnosus GG ATCC 533103 and 37 Lactobacillus strains isolated from traditional fermented milk and pickle (from Xinjiang, Sichuan, and Tibet provinces) were obtained from the Culture and Information Center of Industrial Microorganisms of China Universities, Jiangnan University (CICIM-CU; Wuxi, China), and cultured in de Man, Rogosa, and Sharpe (MRS) broth (Hopebio Co., Qingdao, China) at $37^{\circ} \mathrm{C}$ for $18 \mathrm{~h}$. Helicobacter pylori type strains SS1, 11637, 26695, and 43504 and H. pylori clinical strain C0707 were obtained from CICIM-CU, and H. pylori was cultured on Columbia base agar (Oxoid, Basingstoke, UK) supplemented with $7 \%$ sheep blood and $0.4 \% \mathrm{H}$. pylori selective supplement (Oxoid) at $37^{\circ} \mathrm{C}$ and $5 \% \mathrm{CO}_{2}$.

\section{Acid Tolerance Test}

The acid tolerance test of Lactobacillus strains was carried out by the method of Liong and Shah with modifications (Liong and Shah, 2005; Ding and Shah, 2009). Briefly, MRS broth was adjusted to $\mathrm{pH} 3.0$ with $5 \mathrm{M} \mathrm{HCl}$. Approximately $10^{8} \mathrm{cfu} / \mathrm{mL}$ of each Lactobacillus strain was culture in the acidified MRS broth and incubated at $37^{\circ} \mathrm{C}$ for up to $18 \mathrm{~h}$; absorbance was measured at $600 \mathrm{~nm}\left(\mathbf{A}_{600}\right)$ before and after culture. The survival rates of the lactobacilli at $\mathrm{pH} 2.5$ and 2.0 were determined by the method of Zhou et al. (2007). Briefly, $10^{8} \mathrm{cfu} / \mathrm{mL}$ of each Lactobacillus strain was inoculated into a medium simulating gastric conditions. The medium contained water, $5 \mathrm{~g} / \mathrm{L} \mathrm{NaCl}$, and $3 \mathrm{~g} / \mathrm{L}$ pepsin, and the $\mathrm{pH}$ was adjusted to 2.5 and 2.0 with $\mathrm{HCl}$.

\section{Growth Inhibition Assay}

Cultures of H. pylori were plated on fresh Columbia agar plates without antibiotics $\left(10^{8} \mathrm{cfu}\right.$ per plate), and 6-mm discs were put into the plates with $H$. pylori. Aliquots of $100 \mu \mathrm{L}$ of fresh cultures of the Lactobacillus strains were added into the discs. Plates were incubated for 48 to $72 \mathrm{~h}$ under microaerophilic conditions at $37^{\circ} \mathrm{C}$, and the diameters of the inhibition zones around the wells were measured (Sgouras et al., 2004; Ryan et al., 2008a). Escherichia coli DH5a culture and MRS medium were used as negative controls for in vitro anti- $H$. pylori activity, and metronidazole (0.0005- $0.05 \mathrm{mg} /$ $\mathrm{mL}$ ) was used as a positive control.

\section{Spectrophotometric Autoaggregation Assays}

Autoaggregation abilities were determined and expressed as autoaggregation percentages, and were carried out by the method of Kos et al. (2003) with modifications. Briefly, cells from an 18-h culture of the Lactobacillus strains were prepared as described above. The $\mathrm{A}_{600}$ was adjusted to $0.50 \pm 0.05$ to standardize the number of lactobacilli $\left(10^{7}-10^{8} \mathrm{cfu} / \mathrm{mL}\right)$. Then, the lactobacilli suspensions were incubated in aliquots at $37^{\circ} \mathrm{C}$ and were monitored for different durations $(0,2$, 4,16 , and $24 \mathrm{~h}$ ). The autoaggregation percentage was expressed as follows:

$$
\text { Autoaggregation }(\%)=\left(1-\frac{A_{t}}{A_{0}} \times 100\right) \text {, }
$$

where $A_{t}$ and $A_{0}$ represent $\mathrm{A}_{600}$ of the upper suspensions at different times and total lactobacilli suspensions, respectively.

\section{Coaggregation Assays of $\mathrm{H}$. pylori with Lactobacillus Strains}

The coaggregation test was performed as described by Kos et al. (2003) with modifications. Briefly, Lactobacillus strains and $H$. pylori suspensions were prepared as described in autoaggregation analysis, and equal volumes of cells $\left(10^{7}-10^{8} \mathrm{cfu} / \mathrm{mL}\right)$ of the different Lactobacillus strains and $H$. pylori were mixed and incubated at $37^{\circ} \mathrm{C}$ without agitation. Absorbance was determined for the mixture and for the bacterial suspensions alone. Coaggregation was calculated as follows:

$$
\text { Coaggregation }(\%)=\frac{\left(A_{x}+A_{y}\right)-2 \times A_{m i x}}{A_{x}+A_{y}} \times 100,
$$

where $A_{x}$ and $A_{y}$ represent $\mathrm{A}_{600}$ of the mixed bacterial suspensions at time $0 \mathrm{~h}$, and $A_{m i x}$ represents the $\mathrm{A}_{600}$ of the mixed bacterial suspension at different times.

\section{Bacterial Adhesion to Hydrocarbons}

The bacterial adhesion to hydrocarbons (BATH) test was performed according to the method of Collado et al. (2008) with some modifications. Cells were washed once with PBS and resuspended in the same buffer to an $\mathrm{A}_{600}$ of approximately $0.50 \pm 0.05$ to standardize the number of lactobacilli $\left(10^{7}-10^{8} \mathrm{cfu} / \mathrm{mL}\right)$, and an equal volume of xylene was added. The 2-phase system was thoroughly mixed by vortexing for $5 \mathrm{~min}$. The aqueous phase was removed after $1 \mathrm{~h}$ of incubation at room tem- 
Table 1. Survival rate of selected lactobacilli under the simulated gastric conditions at $\mathrm{pH} 2.0$ for $1 \mathrm{~h}$ and percentage affinity to hydrocarbons

\begin{tabular}{lcc}
\hline Strain & $\begin{array}{c}\text { Survival } \\
\text { rate }(\%)\end{array}$ & $\begin{array}{c}\text { Affinity to } \\
\text { hydrocarbons }(\%)\end{array}$ \\
\hline Lactobacillus rhamnosus GG & $39.58 \pm 1.43^{*}$ & $45.50 \pm 5.38^{*}$ \\
Lactobacillus reuteri C234 & $5.06 \pm 1.38^{*}$ & $8.02 \pm 0.66$ \\
Lactobacillus casei ssp. casei & $0.06 \pm 0.03$ & $39.12 \pm 10.03^{*}$ \\
L. casei Zhang & $0.08 \pm 0.06$ & $47.58 \pm 7.75^{*}$ \\
L. casei BD-II & $0.00 \pm 0.00$ & $-1.23 \pm 0.70$ \\
L. casei 2W & $0.00 \pm 0.00$ & $18.72 \pm 10.32$ \\
Lactobacillus plantarum S3 & $0.81 \pm 0.16$ & $6.24 \pm 4.94$ \\
L. plantarum CZ211-2 & $0.04 \pm 0.01$ & $2.74 \pm 0.66$ \\
L. plantarum DJ201 & $0.00 \pm 0.00$ & $3.29 \pm 0.52$ \\
L. plantarum DJ102-1 & $0.00 \pm 0.00$ & $-4.62 \pm 1.38$ \\
L. plantarum ST-III & $4.93 \pm 1.53^{*}$ & $7.64 \pm 3.38$ \\
L. plantarum ZS2058 & $9.30 \pm 4.05^{*}$ & $18.58 \pm 14.92$ \\
L. plantarum 18 & $0.01 \pm 0.00$ & $1.12 \pm 12.52$ \\
Lactobacillus bulgaricus L24 & $0.00 \pm 0.00$ & $7.19 \pm 1.45$ \\
L. bulgaricus 2-3 & $0.00 \pm 0.00$ & $8.31 \pm 11.79$ \\
Lactobacillus gasseri Chen & $2.25 \pm 1.62^{*}$ & $59.27 \pm 5.91^{*}$ \\
\hline
\end{tabular}

*Significant differences $(P<0.05)$ among all the bacterial strains tested.

perature and its $\mathrm{A}_{600}$ was measured. Affinity to hydrocarbons (hydrophobicity) was reported as the adhesion percentage according to the following formula:

$$
\text { Affinity to hydrocarbons }(\%)=\frac{A_{0}-A}{A_{0}} \times 100 \text {, }
$$

where $A_{0}$ and $A$ are the absorbencies before and after extraction with organic solvents, respectively. Hydrophobicity was calculated as the percentage decrease in the optical density of the original bacterial suspension due to the cells partitioning into a hydrocarbon layer.

\section{Urease Activity Assays}

Urease activity was determined by a modified phenol red method (Sgouras et al., 2004). Briefly, $10 \mu \mathrm{L}$ of fresh lactobacilli or Lactobacillus supernatant was added to $40 \mu \mathrm{L}$ of $H$. pylori cell suspension on a microtiter plate and incubated for $48 \mathrm{~h}$ at $37^{\circ} \mathrm{C}$. Then, $150 \mu \mathrm{L}$ of urease reaction buffer $(20 \%$, wt/vol, urea and $0.012 \%$ phenol red in phosphate buffer with the final $\mathrm{pH}$ adjusted to 6.8) was added to the microtiter plate, and the plate was incubated at $37^{\circ} \mathrm{C}$ for $20 \mathrm{~min}$. The absorbance at $550 \mathrm{~nm}$ was measured with a Sunrise microtiter plate reader (MDC Spectramax, Molecular Devices Inc., Sunnyvale, CA).

\section{Statistical Analysis}

All analyses were conducted in triplicate and the results were statistically analyzed by computing means and standard deviations of the mean. Differences between means of the test and control groups were evaluated by the Dunnett's test, and $P<0.05$ was considered significantly different by one-way ANOVA and SPSS 13.0 (SPSS Inc., Chicago, IL).

\section{RESULTS AND DISCUSSION}

\section{Acid Tolerance Tests}

The $\mathrm{pH}$ in the human stomach ranges from 1 to 4 (Chou and Weimer, 1999). Thirty-eight Lactobacillus strains were tested for tolerance to $\mathrm{HCl}$ at $\mathrm{pH} 3.0$ for $24 \mathrm{~h}$. Twenty-five $(66 \%)$ of the 38 Lactobacillus strains could grow in MRS medium at pH 3.0 (data not shown). Because the essential culture conditions of the human stomach consist of a $\mathrm{pH}(2-3)$ and the presence of pepsin and $\mathrm{NaCl}$, the 25 Lactobacillus strains were inoculated under simulated gastric conditions with $\mathrm{pH} 2.5$ and 2.0 values. The results showed that 16 of 25 lactobacilli could survive under simulated gastric conditions at $\mathrm{pH}$ 2.5 for $3 \mathrm{~h}$ (data not shown), and 10 of 16 lactobacilli could survive under the simulated gastric conditions at $\mathrm{pH} 2.0$ for $1 \mathrm{~h}$ (Table 1). The survival rates of 5 Lactobacillus strains were $>1 \%$, and L. rhamnosus GG exhibited the highest survival rate, approximately $39.58 \%$. The survival rates from the highest to the lowest were in the following order: L. rhamnosus $\mathrm{GG}$, L. plantarum ZS2058, L. reuteri C234, L. plantarum ST-III, and L. gasseri Chen.

Lactobacilli screened for activity against $H$. pylori should survive under simulated gastric conditions. If lactobacilli are capable of surviving for a significant length of time in the stomach, they might be useful in the treatment of gastric disorders (Ryan et al., 2008b). In this study, 10 out of 16 lactobacilli could survive 
Table 2. Ability of 16 Lactobacillus strains to inhibit growth of Helicobacter pylori: the absorbance (optical density, OD, at $600 \mathrm{~nm}$ ) and $\mathrm{pH}$ of the culture medium for each strain after $18 \mathrm{~h}$ of growth and zone of inhibition (ZOI; mm, mean \pm SEM) and urease activity (optical density, $\mathrm{OD}$, at $550 \mathrm{~nm}$ ) of $H$. pylori coculture with lactobacilli supernatants

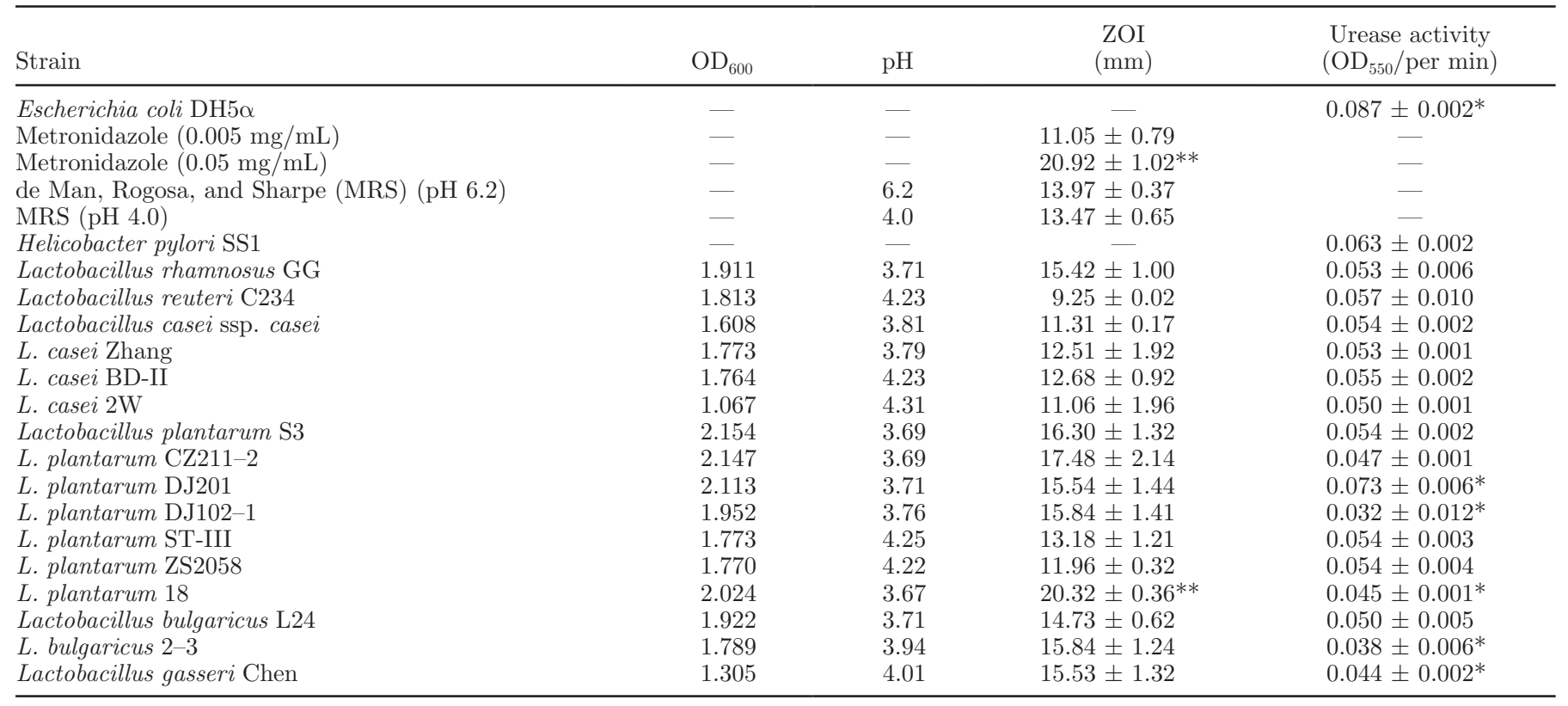

*Significant difference $(P<0.05)$ compared with urease activity of $H$. pylori SS1.

**Significant difference $(P<0.05)$ compared with ZOI of MRS medium inhibition growth of $H$. pylori SS1.

under simulated gastric conditions at $\mathrm{pH} 2.0$ for $1 \mathrm{~h}$, indicating that some lactobacilli could survive in the human stomach.

\section{Growth Inhibition Assay}

The activity of 16 lactobacilli against $H$. pylori SS1 was assessed by using the Oxford plate diffusion assay (Table 2). No inhibition of H. pylori was observed when E. coli DH5 $\alpha$ was used. The fresh MRS medium also exhibited activity against $H$. pylori, and it formed inhibition zones of approximately $14 \mathrm{~mm}$. The inhibition zone diameters of the 16 lactobacilli varied from 9.25 to $20.32 \mathrm{~mm}$. Because the diameters of the inhibition zones formed by 0.005 and $0.05 \mathrm{mg} / \mathrm{mL}$ metronidazole were approximately 12 and $20 \mathrm{~mm}$, respectively, the activity of the 12 lactobacilli against $H$. pylori was equal to that of metronidazole at concentrations between 0.005 and $0.05 \mathrm{mg} / \mathrm{mL}$. The range of $\mathrm{pH}$ in lactobacilli fermentation broths was from 3.6 to 4.3 . Therefore, the antimicrobial activity of lactobacilli may be enhanced by metabolic products including acids. However, no difference in antimicrobial activity was observed between MRS media at $\mathrm{pH} 4.0$ and $\mathrm{pH} 6.2$, which indicated that the main inhibitory effect might not be due to the variation in $\mathrm{pH}$ of the medium.

Probiotics have a proven benefit in reducing the side effects of $H$. pylori therapy. However, the inhibitory ef- fects of lactobacilli on $H$. pylori differ from strain to strain. Using the agar-well diffusion method, Ryan et al. (2008a) showed that Lactobacillus salivarius had high anti-H. pylori activity, and Boyanova et al. (2009) evaluated the activities of 6 strains of Lactobacillus delbrueckii ssp. bulgaricus against $30 \mathrm{H}$. pylori strains and indicated that $L$. delbrueckii ssp. bulgaricus activity was strain-dependent and higher at low $\mathrm{pH}$ than at neutral $\mathrm{pH}$ against H. pylori. Similarly, in the current study, 9 Lactobacillus strains had zones of inhibition $>14 \mathrm{~mm}$, and 1 strain, L. plantarum 18, had significant anti- $H$. pylori activity.

\section{Urease Activity Assays}

Table 2 shows the urease activity of $H$. pylori coculture with 16 Lactobacillus supernatants. The urease activity of 5 strains differed significantly between the cocultures of Lactobacillus supernatants with $H$. pylori and individual $H$. pylori culture $(P<0.05)$. Four Lactobacillus strains showed significant inhibition of the urease activity of H. pylori; however, L. plantarum DJ201 could not inhibit the urease activity of $H$. pylori. Likewise, E. coli DH5a could not inhibit the urease activity of H. pylori. Lactobacillus fermentation broths and viable cells showed good ability to inhibit the urease activity of $H$. pylori, whereas dead cells could not inhibit the activity (data not shown). 
Helicobacter pylori can survive in the acidic environment of the stomach by producing urease, which catalyzes hydrolysis of urea to yield ammonia and elevates the $\mathrm{pH}$ of its environment (Celli et al., 2009). Although $H$. pylori can counteract stomach acid by producing urease, inhibition of urease by some lactobacilli could render H. pylori susceptible to lactic acid (Midolo et al., 1995; Boyanova et al., 2009). By increasing the outer membrane permeability of gram-negative bacteria, lactic acid could improve antibiotic activity (Boyanova et al., 2009). In the present study, the urease activity of $H$. pylori coculture with lactobacillus supernatants, fermentation broths, and viable cells decreased, whereas nonviable cells could not inhibit the activity. It was concluded that lactobacilli could act against $H$. pylori via inhibition of urease activity.

However, many substances have been reported to have anti- $H$. pylori activity, including lactic acid, bacteriocins, peroxides, proteinases, exopolysaccharides, and viable cells. Some studies have suggested that the production of relatively large amounts of lactate by lactobacilli is an inhibitory factor for H. pylori (Midolo et al., 1995; Aiba et al., 1998). Lactic acid, in addition to its antimicrobial effect resulting from lowering of $\mathrm{pH}$, could inhibit H. pylori urease. However, other studies have demonstrated that lactobacilli exert inhibitory effects on $H$. pylori by lactic acid- and $\mathrm{pH}$-independent mechanisms (Kim et al., 2003; Sgouras et al., 2004), and this inhibitory activity could be associated with the viable cells (Ryan et al., 2008a) and antimicrobial substances such as bacteriocins, peroxides, proteinases, exopolysaccharides, and cell wall components. Therefore, inhibition of lactobacilli against $H$. pylori could be attributed to inhibition of urease activity, the exposure of $H$. pylori to an acidic environment, or competitive growth with $H$. pylori.

\section{Spectrophotometric Autoaggregation and Coaggregation Assays of $H$. pylori and Lactobacilli}

The autoaggregation properties of lactobacilli were examined over a period of $24 \mathrm{~h}$ at $37^{\circ} \mathrm{C}$ by absorbance measurements at $600 \mathrm{~nm}$; the results are shown in Table 3. In general, most lactobacilli exhibited a strong autoaggregating phenotype, and autoaggregation rates increased with incubation time. The highest autoaggregation rates were obtained with $L$. bulgaricus $2-3$ and L. gasseri Chen. Lactobacillus rhamnosus GG, L. casei BD-II, and L. plantarum DJ201 also showed high autoaggregation rates $(>45 \%)$ after $24 \mathrm{~h}$ of incubation.

Coaggregation abilities were tested using an indicator strain of $H$. pylori at different times, and the results are shown in Table 3 . The coaggregation rates increased

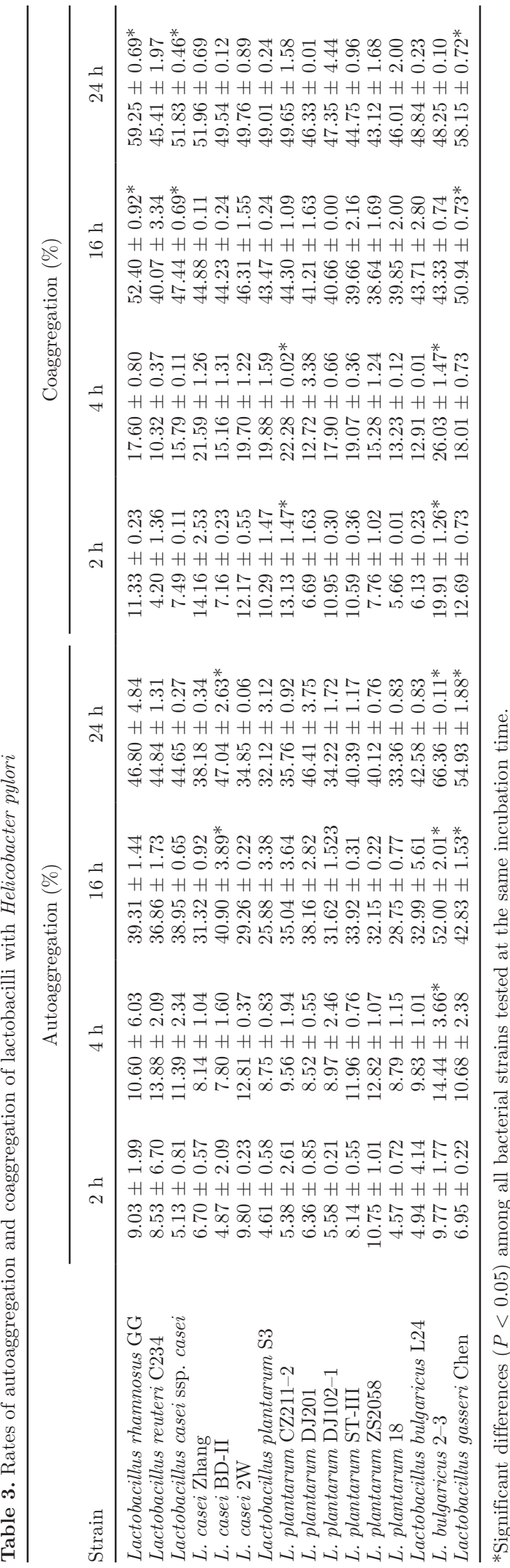

Journal of Dairy Science Vol. 93 No. 12, 2010 

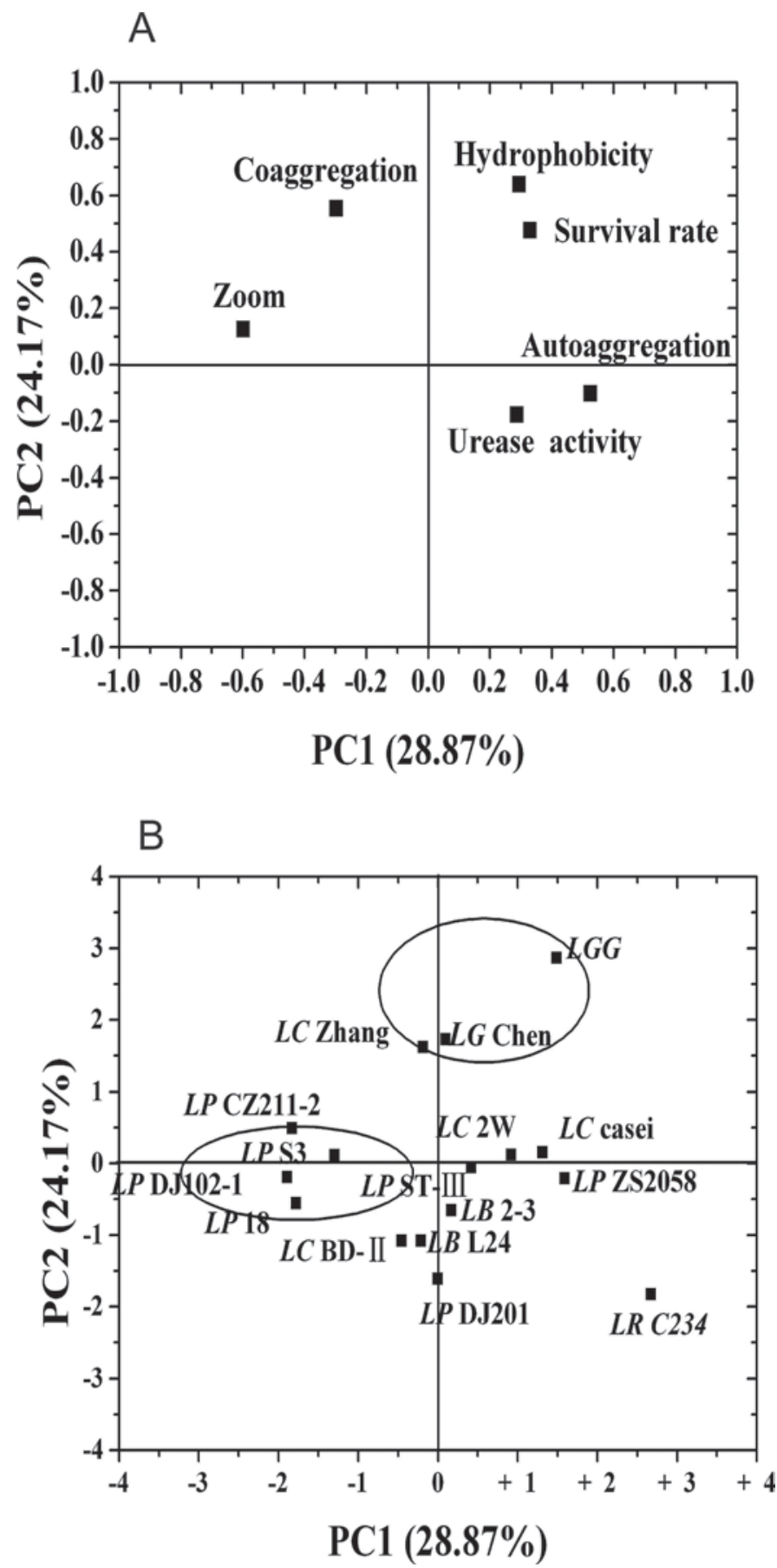

Figure 1. Graphical representation of the principal component analysis of lactobacilli properties from the trained panel showing principal component (PC)1 versus PC2: (A) factor loadings; (B) factor scores. $L P=$ Lactobacillus plantarum; $L C=$ Lactobacillus casei $L B=$ Lactobacillus bulgaricus; $L R=$ Lactobacillus reuteri; $L G=$ Lactobacillus gasseri; and $L G G=$ Lactobacillus rhamnosus $\mathrm{GG}$. with the incubation time, and the highest coaggregation percentages were obtained with $L$. rhamnosus GG $(59.3 \%)$ and $L$. gasseri Chen $(58.2 \%)$ after $24 \mathrm{~h}$ of incubation. However, no significant differences $(P>0.05)$ were found between the 2 strains.

\section{Bacterial Adhesion to Hydrocarbons}

The adhesion percentages of lactobacilli to xylene are shown in Table 1, and the percentage affinity to hydrocarbons (hydrophobicity) ranged between 0.0 and $59.3 \%$ for the test strains. The most hydrophobic strain was L. gasseri Chen (59.3\%); L. rhamnosus GG and $L$. casei Zhang also had high hydrophobicities, which were 45.5 and $47.6 \%$, respectively. In general, most lactobacilli tested showed high hydrophobicity but $L$. casei BD-II and L. plantarum DJ102-1 had low hydrophobicity (below zero).

\section{Principal Component Analysis}

Principal component analysis (PCA) was performed to investigate the correlation among the various properties of Lactobacillus (aggregation, coaggregation, and hydrocarbons) and the ability to inhibit $H$. pylori growth and urease activity and tolerance to acids. The results, as seen in Figure 1a, were defined by 4 principal components (PC): (1) the first (PC1), which explained $28.87 \%$ of the variance in the model, was characterized by inhibition zone; (2) the second (PC2) explained $24.17 \%$ of the variance in the model and was characterized by coaggregation and hydrophobicity; (3) the third (PC3) explained $19.40 \%$ of the variance in the model and was characterized by urease activity and aggregation; and (4) the fourth (PC4) explained 14.54\% of the variance in the model and was characterized by the survival rate in acids.

Aggregation properties, together with the ability to coaggregate with the potential pathogen, could be used for preliminary selection of probiotic bacteria against the pathogen (Vlkova et al., 2008). Aggregation plays an important role in the formation of biofilms. Although coaggregation is not indicative of biofilm formation, it may play an important role in eliminating pathogens from the gastrointestinal tract (Todorov et al., 2008). Cell surface hydrophobicity is a nonspecific interaction between microbial cells and host cells. Bacterial cells with a high hydrophobicity usually form strong interactions with mucosal cells. Therefore, multiple properties of Lactobacillus may affect its inhibitory activity against H. pylori; however, the exact mechanism of inhibition against $H$. pylori should be further studied. 
Table 4. The inhibition of the growth of Helicobacter pylori standard and clinical strains by Lactobacillus plantarum 18 and Lactobacillus gasseri Chen

\begin{tabular}{lcccc}
\hline & \multicolumn{4}{c}{ Zone of inhibition $(\mathrm{mm}$; mean \pm SEM) } \\
\cline { 2 - 5 } Strain & $\begin{array}{c}\text { H. pylori } \\
43504\end{array}$ & $\begin{array}{c}\text { H. pylori } \\
11637\end{array}$ & $\begin{array}{c}\text { H. pylori } \\
26695\end{array}$ & $\begin{array}{c}\text { H. pylori } \\
\text { C0707 }\end{array}$ \\
\hline L. plantarum 18 & $14.79 \pm 0.58^{*}$ & $13.16 \pm 2.24$ & $13.95 \pm 1.36^{*}$ & $16.25 \pm 1.10^{*}$ \\
L. gasseri Chen & $14.58 \pm 0.60^{*}$ & $12.14 \pm 1.78$ & $12.61 \pm 1.66$ & $16.24 \pm 1.89^{*}$ \\
MRS & $11.49 \pm 0.93$ & $10.95 \pm 1.28$ & $10.44 \pm 0.56$ & $12.73 \pm 0.54$ \\
\hline
\end{tabular}

*Within-column significant difference $(P<0.05)$ compared with zone of inhibition of de Man, Rogosa, and Sharpe (MRS) medium.

As seen in Figure 1b, L. plantarum 18, L. plantarum DJ102-1, L. plantarum S3, and L. plantarum CZ211-2 had the highest contribution to PC1, whereas $\mathrm{PC} 2$ was more correlated with L. rhamnosus GG, L. gasseri Chen, and $L$. casei Zhang. The characteristics of $L$. gasseri Chen were the most similar to those of L. rhamnosus GG, which inhibited H. pylori, and these findings suggested that L. gasseri Chen could be used as a potential probiotic against $H$. pylori. Lactobacillus plantarum 18 had the largest zone of inhibition and markedly reduced the urease activity of $H$. pylori. Therefore, according to PCA and the ability of inhibition growth of H. pylori, L. plantarum 18 and L. gasseri Chen showed potential against $H$. pylori.

To further evaluate the activities of $L$. plantarum 18 and L. gasseri Chen against other strains of H. pylori, the inhibition abilities of these 2 lactobacilli on the growth of H. pylori were tested (Table 4). Although all $4 \mathrm{H}$. pylori strains were inhibited by these 2 lactobacillus strains, the degree of inhibition varied between $H$. pylori strains.

Therefore, L. plantarum 18 and L. gasseri Chen showed great potential for applications in prevention and treatment of H. pylori infection. Further research is required to investigate the mechanisms of the selected lactobacilli underlying the activity against $H$. pylori infection in vitro.

\section{CONCLUSIONS}

This study utilized a multi-step method to screen lactobacilli against $H$. pylori, and the results indicated that L. plantarum 18 and L. gasseri Chen had potential capacity to inhibit the growth and urease activity of H. pylori. Moreover, these 2 strains survived under the simulated gastric conditions. Therefore, L. plantarum 18 and L. gasseri Chen show potential anti-Helicobacter pylori activity in vitro, although further research is needed to verify the activities of these strains against H. pylori.

\section{ACKNOWLEDGMENTS}

This work was supported by the National Natural Science Foundation of China (No. 30871952 and 20836003), the National Science \& Technology Pillar Program in the Eleventh Five-year Plan Period (2009BADC1B02, 2009BADB9B05), the National High Technology Research and Development Program of China (2010AA10Z302), the 111 project B07029, and the Researcher Program of Jiangsu Province of China. The authors also thank Xinpeng Li (Shangdong University, China), Neng Li (Fujian Medical University, China), and Chaoling Luo (Shanghai Linc-Bio Science Co. Ltd., China) for technical assistance during the study.

\section{REFERENCES}

Aiba, Y., N. Suzuki, A. M. Kabir, A. Takagi, and Y. Koga. 1998. Lactic acid-mediated suppression of Helicobacter pylori by the oral administration of Lactobacillus salivarius as a probiotic in a gnotobiotic murine model. Am. J. Gastroenterol. 93:2097-2101.

Armuzzi, A., F. Cremonini, F. Bartolozzi, F. Canducci, M. Candelli, V. Ojetti, G. Cammarota, M. Anti, A. De Lorenzo, P. Pola, G. Gasbarrini, and A. Gasbarrini. 2001. The effect of oral administration of Lactobacillus GG on antibiotic-associated gastrointestinal side-effects during Helicobacter pylori eradication therapy. Aliment. Pharmacol. Ther. 15:163-169.

Bergonzelli, G. E., D. Granato, R. D. Pridmore, L. F. Marvin-Guy, D. Donnicola, and I. E. Corthesy-Theulaz. 2006. GroEL of Lactobacillus johnsonii La1 (NCC 533) is cell surface associated: Potential role in interactions with the host and the gastric pathogen Helicobacter pylori. Infect. Immun. 74:425-434.

Boyanova, L., M. Stephanova-Kondratenko, and I. Mitov. 2009. Anti-Helicobacter pylori activity of Lactobacillus delbrueckii subsp bulgaricus strains: Preliminary report. Lett. Appl. Microbiol. 48:579-584.

Celli, J. P., B. S. Turner, N. H. Afdhal, S. Keates, I. Ghiran, C. P. Kelly, R. H. Ewoldt, G. H. McKinley, P. So, S. Erramilli, and R. Bansil. 2009. Helicobacter pylori moves through mucus by reducing mucin viscoelasticity. Proc. Natl. Acad. Sci. USA 106:1432114326.

Chou, L. S., and B. Weimer. 1999. Isolation and characterization of acid- and bile-tolerant isolates from strains of Lactobacillus acidophilus. J. Dairy Sci. 82:23-31.

Collado, M. C., A. Gonzalez, R. Gonzalez, M. Hernandez, M. A. Ferrus, and Y. Sanz. 2005. Antimicrobial peptides are among the antagonistic metabolites produced by Bifidobacterium against $\mathrm{He}$ licobacter pylori. Int. J. Antimicrob. Agents 25:385-391. 
Collado, M. C., J. Meriluoto, and S. Salminen. 2008. Adhesion and aggregation properties of probiotic and pathogen strains. Eur. Food Res. Technol. 226:1065-1073.

Dhar, S. K., R. K. Soni, B. K. Das, and G. Mukhopadhyay. 2003. Molecular mechanism of action of major Helicobacter pylori virulence factors. Mol. Cell. Biochem. 253:207-215.

Ding, W. K., and N. P. Shah. 2009. An improved method of microencapsulation of probiotic bacteria for their stability in acidic and bile conditions during storage. J. Food Sci. 74:M53-M61.

Goodwin, C. S. 1997. Helicobacter pylori gastritis, peptic ulcer, and gastric cancer: Clinical and molecular aspects. Clin. Infect. Dis. 25:1017-1019.

Gotteland, M., M. Andrews, M. Toledo, L. Munoz, P. Caceres, A Anziani, E. Wittig, H. Speisky, and G. Salazar. 2008. Modulation of Helicobacter pylori colonization with cranberry juice and Lactobacillus johnsonii La1 in children. Nutrition 24:421-426.

Graham, D. Y. 1997. Helicobacter pylori infection in the pathogenesis of duodenal ulcer and gastric cancer: a model. Gastroenterology 113:1983-1991.

Kabir, A. M., Y. Aiba, A. Takagi, S. Kamiya, T. Miwa, and Y. Koga 1997. Prevention of Helicobacter pylori infection by lactobacilli in a gnotobiotic murine model. Gut 41:49-55.

Kim, T. S., J. W. Hur, M. A. Yu, C. I. Cheigh, K. N. Kim, J. K Hwang, and Y. R. Pyun. 2003. Antagonism of Helicobacter pylori by bacteriocins of lactic acid bacteria. J. Food Prot. 66:3-12.

Kos, B., J. Suskovic, S. Vukovic, M. Simpraga, J. Frece, and S. Matosic. 2003. Adhesion and aggregation ability of probiotic strain Lactobacillus acidophilus M92. J. Appl. Microbiol. 94:981-987.

Lee, S. Y., Y. W. Shin, and K. B. Hahm. 2008. Phytoceuticals: Mighty but ignored weapons against Helicobacter pylori infection. J. Dig. Dis. 9:129-139.

Lesbros-Pantoflickova, D., I. Corthésy-Theulaz, and A. L. Blum. 2007. Helicobacter pylori and probiotics. J. Nutr. 137:812-818.

Liong, M. T., and N. P. Shah. 2005. Acid and bile tolerance and cholesterol removal ability of lactobacilli strains. J. Dairy Sci. $88: 55-66$

Lorca, G. L., T. Wadstrom, G. F. Valdez, and A. Ljungh. 2001. Lactobacillus acidophilus autolysins inhibit Helicobacter pylori in vitro. Curr. Microbiol. 42:39-44.

Midolo, P. D., J. R. Lambert, R. Hull, F. Luo, and M. L. Grayson. 1995. In vitro inhibition of Helicobacter pylori NCTC 11637 by organic acids and lactic acid bacteria. J. Appl. Bacteriol. 79:475479 .

Ouwehand, A. C., E. Isolauri, P. V. Kirjavainen, S. Tolkko, and S. J. Salminen. 2000. The mucus binding of Bifidobacterium lactis Bb12 is enhanced in the presence of Lactobacillus GG and Lact. delbrueckii ssp. bulgaricus. Lett. Appl. Microbiol. 30:10-13.

Pinchuk, I. V., P. Bressollier, B. Verneuil, B. Fenet, I. B. Sorokulova, F Megraud, and M. C. Urdaci. 2001. In vitro anti-Helicobacter pylor activity of the probiotic strain Bacillus subtilis 3 is due to secretion of antibiotics. Antimicrob. Agents Chemother. 45:3156-3161.

Ryan, K. A., P. Daly, Y. Li, C. Hooton, and P. W. O'Toole. 2008a. Strain-specific inhibition of Helicobacter pylori by Lactobacillus salivarius and other lactobacilli. J. Antimicrob. Chemother. 61:831-834.

Ryan, K. A., T. Jayaraman, P. Daly, C. Canchaya, S. Curran, F. Fang, E. M. Quigley, and P. W. O'Toole. 2008b. Isolation of lactobacilli with probiotic properties from the human stomach. Lett. Appl. Microbiol. 47:269-274.

Sakamoto, I., M. Igarashi, K. Kimura, A. Takagi, T. Miwa, and Y. Koga. 2001. Suppressive effect of Lactobacillus gasseri OLL 2716 (LG21) on Helicobacter pylori infection in humans. J. Antimicrob. Chemother. 47:709-710.

Sgouras, D., P. Maragkoudakis, K. Petraki, B. Martinez-Gonzalez, E. Eriotou, S. Michopoulos, G. Kalantzopoulos, E. Tsakalidou, and A. Mentis. 2004. In vitro and in vivo inhibition of Helicobacter pylori by Lactobacillus casei strain Shirota. Appl. Environ. Microbiol. 70:518-526.

Todorov, S. D., M. Botes, C. Guigas, U. Schillinger, I. Wiid, M. B. Wachsman, W. H. Holzapfel, and L. M. Dicks. 2008. Boza, a natural source of probiotic lactic acid bacteria. J. Appl. Microbiol. 104:465-477.

Vlkova, E., V. Rada, M. Smehilova, and J. Killer. 2008. Auto-aggregation and co-aggregation ability in bifidobacteria and clostridia. Folia Microbiol. (Praha) 53:263-269.

Zhou, X. X., Y. J. Pan, Y. B. Wang, and W. F. Li. 2007. In vitro assessment of gastrointestinal viability of two photosynthetic bacteria, Rhodopseudomonas palustris and Rhodobacter sphaeroides. J. Zhejiang Univ. Sci. B 8:686-692. 